\title{
The clinical utility of preformed C1q-binding donor-specific anti-HLA antibodies in kidney transplantation
}

\author{
Sua Lee ${ }^{1}$, Chul Woo Yang ${ }^{2}$, Byung Ha Chung ${ }^{2}$ \\ ${ }^{1}$ Division of Nephrology, Department of Internal Medicine, Seoul National University Hospital, Seoul, Korea \\ ${ }^{2}$ Division of Nephrology, Department of Internal Medicine, Seoul St. Mary's Hospital, College of Medicine, The Catholic University of Korea, Seoul, Korea
}

Background: The role of C1q-binding donor-specific antibody (DSA) in antibody-mediated rejection (AMR) is considered important. De novo C1q-binding DSA is well known as an associated factor for increased risk of AMR and graft loss. However, the impact of preformed C1q-binding DSA is not yet clear. We investigated the clinical utility of identification of preformed C1q-binding DSA for predicting graft outcomes in kidney transplantation (KT).

Methods: From December 2016 to December 2019, 373 recipients underwent living-donor KT at St. Mary's Hospital. If the result of panel reactive antibody was positive in the pre-transplant, DSA and C1q-binding DSA were performed using Luminex single antigen bead assay. According to the presence of C1q-binding DSA, recipients were classified as C1q-positive and C1q-negative groups. Primary outcome was biopsy-proven acute AMR.

Results: Of 373, 75 recipients (20.1\%) had preformed DSA. Among them, 16 recipients (4.3\%) had preformed C1q-binding DSA. C1q-positive group had more positive panel reactive antibody (PRA) class II and DSA class II with statistical significance ( $P=0.036$ and $\mathrm{P}=0.050$, respectively). DSA class II mean fluorescence intensity in C1q-positive group was significantly higher than in C1q-negative group (median [interquartile range], 13,796 [10,746-22,883] vs. 5,055 [1,247-7,697]; $P<0.001)$. The incidence of acute rejection in C1q-positive group was significantly higher than in $\mathrm{C} 1 \mathrm{q}$-negative group, especially acute $\mathrm{AMR}(\mathrm{P}=0.037$ and $P=0.040$, respectively). In Kaplan-Meier analysis, the cumulative incidence of acute AMR in C1q-positive group was significantly

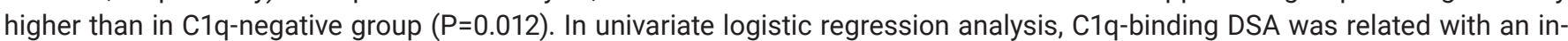
creased risk of acute AMR (hazard ratio, 3.81; $P=0.023$ ).

Conclusions: Preformed C1q-binding DSA would be associated with an increased risk of acute AMR. Surveillance, such as protocol allograft biopsy, can help to detect acute AMR early in recipients with preformed C1q-binding DSA.

Corresponding author: Sua Lee

E-mail: soulmysoul27@naver.com

(C) The Korean Society for Transplantation

This is an Open Access article distributed under the terms of the Creative Commons Attribution Non-Commercial License (http://creativecommons.org/licenses/by-nc/4.0/) which permits unrestricted non-commercial use, distribution, and reproduction in any medium, provided the original work is properly cited. 